\title{
The Virtual Playground: an Educational Virtual Reality Environment for Evaluating Interactivity and Conceptual Learning
}

\author{
Maria Roussou $^{1}$, Martin Oliver ${ }^{2}$, Mel Slater ${ }^{1,3}$ \\ ${ }^{1}$ Department of Computer Science, University College London, UK \\ ${ }^{2}$ London Knowledge Lab, Institute of Education, UK \\ ${ }^{3}$ ICREA - Universitat Politècnica de Catalunya, Spain
}

Tel. +44 2076793664

Fax +44 2073871317

m.roussou@cs.ucl.ac.uk

http://www.cs.ucl.ac.uk/staff/M.Roussou/research/

\begin{abstract}
The research presented in this paper aims at investigating user interaction in immersive virtual learning environments (VLEs), focusing on the role and the effect of interactivity on conceptual learning. The goal has been to examine if the learning of young users improves through interacting in (i.e. exploring, reacting to, and acting upon) an immersive virtual environment (VE) compared to non interactive or non-immersive environments. Empirical work was carried out with more than 55 primary school students between the ages of 8 and 12, in different between-group experiments: an exploratory study, a pilot study, and a large-scale experiment. The latter was conducted in a virtual environment designed to simulate a playground. In this 'Virtual Playground', each participant was asked to complete a set of tasks designed to address arithmetical 'fractions' problems. Three different conditions, two experimental virtual reality (VR) conditions and a non-VR condition, that varied the levels of activity and interactivity, were designed to evaluate how children accomplish the various tasks. Pre-tests, post-tests, interviews, video, audio, and log files were collected for each participant, and analyzed both quantitatively and qualitatively. This paper presents a selection of case studies extracted from the qualitative analysis, which illustrate the variety of approaches taken by children in the VEs in response to visual cues and system feedback. Results suggest that the fully interactive VE aided children in problem solving but did not provide as strong evidence of conceptual change as expected; rather, it was the passive VR environment, where activity was guided by a virtual robot, that seemed to support student reflection and recall, leading to indications of conceptual change.
\end{abstract}

Keywords: Virtual learning environments, Interactivity, Conceptual

Learning, Evaluation 


\section{Introduction}

In the past two decades, immersive Virtual Reality (VR) has attracted the attention of many researchers and educators who predicted that VR would have considerable impact on the way that learning and teaching is conducted. However, widespread uptake has yet to become apparent and, despite the successful research efforts undertaken, we still know little about what exactly constitutes an effective Virtual Learning Environment (VLE). Hence, more recently, certain research efforts have turned to the empirical study of the influence of some of the distinctive characteristics of VR, such as immersion [] and presence [][], and whether these can or cannot support conceptual learning.

In this research we examine the dimension of interactivity in a Virtual Environment (VE) and, in particular, its potential and limitations for learning. Interactivity is undoubtedly one of the key elements of a VR experience. By interactivity we mean the ability to freely move around a virtual environment, to experience it "first-hand" and from multiple points of view, to modify its elements, to control parameters, or to respond to perceived affordances, environment cues, and system feedback ${ }^{1}$. Studies on the use of VR for training have shown that such activity can be effective, for example, for spatial knowledge acquisition and recall in training []. Interaction and feedback have also often been linked to presence, indicating that user control over the environment was important for the experience of presence [], as was the amount of body movement []. Other studies concluded that the extent to which students were able to control the VE made a greater difference to what they learned than if the system was immersive or not [][].

Despite these findings, little systematic research has concentrated on examining interactivity in relation to learning; hence there is no clear evidence that interactivity alone can bring "added value" to learning,

\footnotetext{
${ }^{1}$ Most of the attempts to define interactivity recognize gradations of activity, both in the physical (kinesthetic) and the intellectual sense. For the purposes of this research, we adopt the general framework proposed by Pares and Pares [], where interactivity in a VE is classified as explorative (involving, in practice, spatial navigation), manipulative (the manipulation of parameters and elements of the VE), and contributive (the ability to alter the system of operation itself).
} 
especially for children. We believe that the activity between the user and the virtual environment may be a defining component in inducing conceptual change and certainly one that is worth examining further. In the following sections, we describe our methodology, the VEs designed, and the empirical work that was carried out in order to examine the effect of interactivity in VEs on conceptual learning, in other words, on the deeper, transferable understandings of abstract knowledge [].

\section{Previous studies of immersive VLEs}

A number of educational VR projects have been developed throughout the years, ranging from research projects conducted in academic laboratory environments to projects that have been applied in formal [] and informal educational settings [][], with a goal to apply and evaluate the potential of virtual reality as a medium for educating students. Many of the early educational VR projects were developed especially for head-mounted display systems (HMDs) whilst the later projects started exploring the use of the physical space along with the virtual by employing projection-based displays (CAVEs) and, more recently, Mixed Reality and Augmented Reality setups [].

A large part of this educational research has been focused on science education, as in the NewtonWorld and MaxwellWorld ScienceSpace projects [], which set out to explore the kinematics and dynamics of motion, electrostatic forces and other physics concepts. The initial formative evaluation reports on learners' engagement, surprise and understanding of the alternative representations of the concepts provided in the ScienceSpace worlds []. Multisensory cues, multimodal interaction, and the introduction of multiple new representations were believed to have helped students develop correct mental models of the abstract material. However, in terms of interactivity, other than navigation and pick-and-place activity, the worlds could not be dynamically altered through the learner's participation.

The Virtual Reality Roving Vehicle (VRRV) project [] and the summer camp programs in VR for students [], initiated by the HIT Lab, focused on "worldbuilding" activity, where students conceived and created the objects of their 
own virtual worlds by using 3D modeling software on desktop computers, which they then experienced in an immersive environment. Similarly, a projection-based display (a CAVE®) was used to display the results of students' model building activity in the Virtual Solar System (VSS) project, an experimental undergraduate astronomy course in which students built models of the solar system in order to learn about astronomical phenomena []. In both cases, student activity involved mainly creating the virtual world rather than interacting with one.

The NICE project was an early interactive virtual reality learning environment that provided young children with a fantasy world in which they could collaboratively cultivate a virtual garden []. In the study of children's behaviour in the NICE environment, interactivity, identified with control over the environment, scored as the most significant motivational component of the learning experience. Giving one child control meant that the child with control tended to be more engaged with the educational content, resulting in a tendency to learn more; however this "measurement" of learning emerged from exploratory observation that looked at general aspects rather than formal processes through which specific conclusions about learning could be drawn. Lessons learned from the NICE project, helped to focus and form the design of the Round Earth Project [] so that the learning domain was carefully selected to focus on a problem proven to be difficult with children. The Round Earth Project investigated how virtual reality could be used to help teach young children that the Earth is spherical when their everyday experiences tell them it is flat. VR was used as part of a larger strategy to create an alternative cognitive starting point where this concept could be established on its own before it was brought into contact with the learner's past experiences []. Further projects by the same group focused on investigating the effectiveness of virtual environments as simulated data collection environments for children engaged in inquiry-based science learning activities.

A study that explored interactivity in the context of geometry teaching with diagrammatic representations, focused on the comparison between different graphical representations of the concept of stereographic projection and the 
effect that the addition of various interactive properties might have on the learning goal []. The results led to the conclusion that just adding interactivity did not seem to increase the efficiency of the learning environment since the interactive $3 \mathrm{D}$ environment did not seem to provide the expected learning gains. However, it was noted that the study was exploratory and additional investigation was required, since learning seemed to be affected by a complex interaction of representation properties, task demands, and within-subject factors.

To summarize, VR projects developed for educational purposes have either not provided the analytical evidence to demonstrate learning as a result of interaction with the environment or, where an educational impact was perceived, there is no explanation of which forms of interactivity are effective. More importantly, the role of interactivity within learning has not been the focus of any of the evaluations carried out as such. Hence, the research question that emerges is how interactivity in a virtual learning environment can influence learning. To provide answers to this question, we first need to address how this can be studied. In the next sections, we describe the design of our studies and the virtual environments created to support them.

\section{Defining a methodology for study and analysis}

Since our goal is to study learning as a result of the learner's interaction with a virtual environment, a learning task had to be specified and an interactive virtual environment built with enough features as to invoke the multiple levels of interactivity found in VR applications []. Our first idea, which was developed with consultation from supportive math and science teachers, was to create a task where the participant had to build a temple by identifying and assembling its various parts. As an idea, the construction of a temple is advantageous because it encompasses an inherently activity-rich process, so it formed the basis for our exploratory studies.

A set of exploratory studies was carried out with children between 8 and 12 years old. The children were asked to complete tasks involving the assembly of ancient columns from parts in an immersive stereoscopic VR system (a 
CAVE®-like display) using a 3D joystick device with buttons for interaction. The learning goal was to understand the differences between columns of different order (Doric and Ionian) and symmetry. The tasks included selection, comparison, and resizing of the column parts in order to fit them onto their correct bases. Since these studies were exploratory, we followed a qualitative approach based on observation (aided by a think-aloud protocol) and informal interviews with the children. We observed the children's activity in the VE and looked for the following different occurrences of learning for the purpose of analyzing our data:

- Conceptual change, where participants revise their conceptions or change their interpretation of something.

- Additive knowledge, where participants have added to what they have already experienced, as long as this involves some kind of reinterpretation of previous action rather than just the accumulation of information.

- Changes in behaviour.

Our method of analysis draws on []: we reviewed the video of the sessions and identified various points where interesting interactions seemed to occur. We chose to focus on moments in time where participants made a statement that indicated they had changed their conception or where we could conclude things from our observation of the participant's behaviour in the environment. The organizational framework of Activity Theory [] provided us with the conceptual vocabulary to help interpret these points qualitatively. Our findings indicated three kinds of instances where learning seemed to take place: learning about the system as a result of technical problems, learning caused by (unintentional) observer intervention and, to a lesser extent, learning arising from system feedback. The latter is what we were most interested in, since they involved interaction between the learner and the digital environment without human mediation. We thus focused on excerpts where such instances provoking internal contradictions leading to conceptual change seemed to occur. These caused the participants to change their behaviour as well as revise their rules and conceptions, triggered by the rules set out by the system. The participants' observation of the system's rules guided them in evaluating their actions, assessing for themselves the 
contradiction within the system and resolving it in order to achieve the objective.

To make the analytical methodology clearer, let us look at the example of 10 year-old John. John had started constructing a column from the "capital" (the top part of the column), which he placed in the air and then begun building downwards by placing each one of the drums underneath. He had managed to squeeze the last drum under the others and attempted to pick up the column base. The VE was not programmed to provide any explicit feedback; however, it was designed with certain features that provided intrinsic feedback, such as the fact that the column bases could not be moved. This was the only type of feedback that represented the system's interactive capabilities and which implicitly aided John in changing his course of action.

1. Observer: How do you see that this piece goes at the bottom rather than the top?

2. John: It's the last piece.

3. Observer: How do you know that it is the last piece?

4. John: Because I put that one [showing the bottom last column drum] and saw that there is no other one that fits below it... Anyway, you can tell it's the last piece.

5. John: [trying to pick up the last piece and realizing that it doesn't move] It is glued on the floor...

6. Observer: Why would it be glued on the floor?

7. John: [thinks for a moment] ...Oh! So that I can put the other pieces here.

He then took apart the column he had constructed in the air and began constructing it piece by piece on top of the base by reversing the sequence in which he was placing the column drums until he reached the capital. The "Oh!" is the "Eureka" moment that both triggers his change in behaviour and indicates a change in his conceptions (Figure 1). Furthermore, in the tasks that followed, John identified the bases immediately, having remembered from this first task that the bases do not move, and started constructing the columns from the bottom working up. 

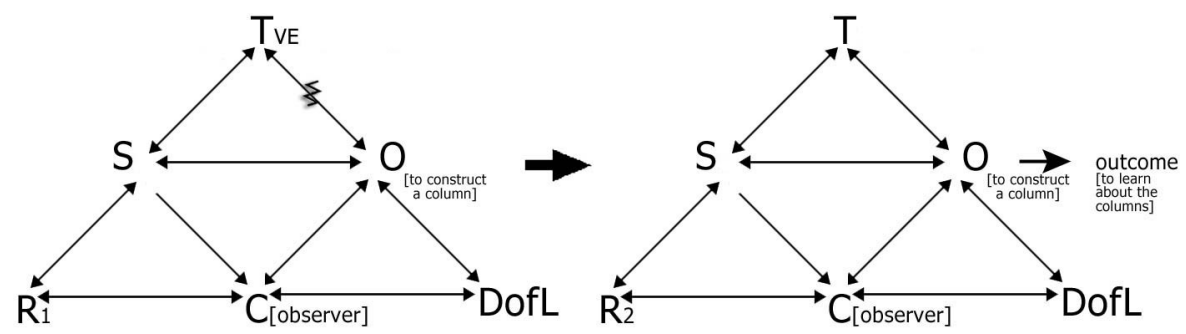

Figure 1. An Activity System illustrating a breakdown between the tool (the VE) and the participant's (John's) goal (O - Object), which is resolved by a revision of the Rules (from R1 to R2).

For a detailed analysis of these exploratory studies using the Activity Theory framework, see [].

Overall, these case studies helped in clarifying issues concerning the methodology for working with children for this problem, while acting as a test bed for the application of the analytical framework. They also allowed shortcomings of the task to be identified; the observed learning outcomes indicated that the learning goal of the tasks (i.e. to learn about the order and symmetry of ancient columns) was not easily quantifiable and did not provide enough opportunities for conceptual learning to occur and, consequently, to be assessed. This led to a re-design of the study, which required the design of a different virtual environment, as discussed in the following section.

\section{The design of an interactive VE to support conceptual learning}

It became apparent that the column construction activity did not provide enough opportunities for conceptual challenge and could not be easily linked to the everyday life and interests of today's children between 8 and 12 years old. Therefore, a different learning domain was chosen that would allow us to exploit the capabilities of the VR medium in visualizing abstract and difficult conceptual learning problems and providing feedback. In order to examine "interactivity", it was decided that varied levels of control over the parameters of the system should be provided through an experimental VE in which children would be asked to complete constructivist tasks that are designed as arithmetical fraction problems. Fractions were chosen as the learning topic due to the difficulty that primary school students have in understanding and 
connecting them to real-world situations []. In other words, fractions lend themselves to designing learning tasks that are, at the same time, conceptually difficult, abstract enough to justify representation via a VR simulation of a real-world situation, and can allow for a kind of varied and incremental interactive treatment.

\subsection{The conceptual learning problem: representing fractions}

Research has shown that students begin to construct a deeper understanding of fractions when these are represented in a variety of ways and when there are explicit linkages to everyday life and familiar situations involving their use. Elementary-school children's difficulties in learning fractions and understanding their representations have been well documented [] while a number of educational technology research projects and products have been developed on this topic [] []. Traditionally, fractions have been represented with a formal symbolic system (for example " $1 / 3$ "), which essentially is an "artificial" construct used for performing arithmetical operations and learning fractions in school. To facilitate understanding of fractions, educators have been using various means and methods to teach them, such as $2 \mathrm{D}$ pictorial representations (the "pie" metaphor), manipulative models (rods, arithmetic blocks, bars, number lines, paper folding exercises, and others), and "real world" story scenarios.
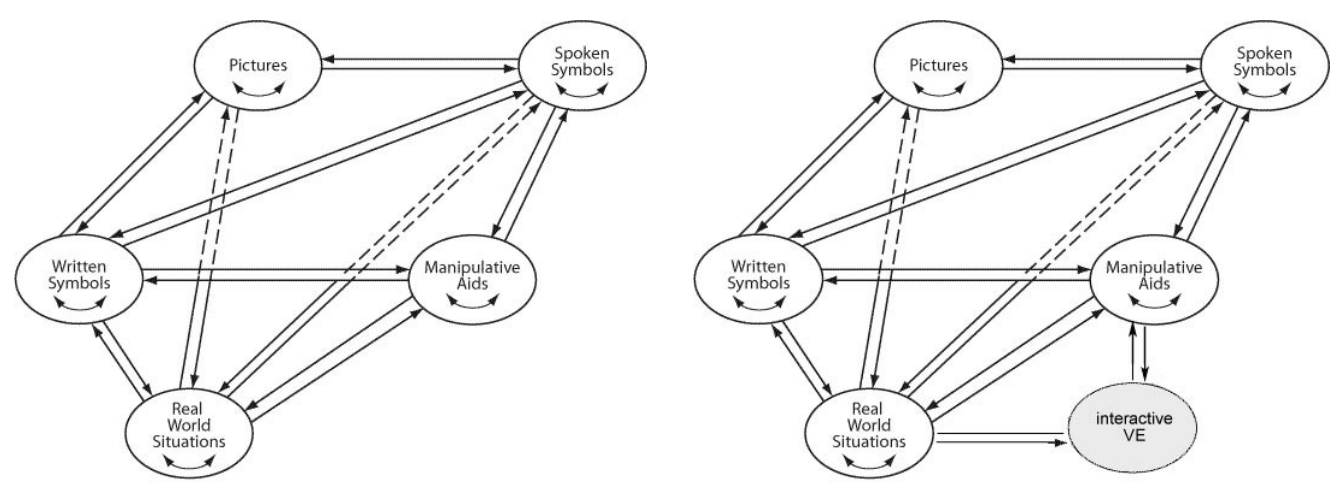

Figure 2. Lesh's translational model (left) illustrates the five distinct types of representing mathematical ideas (fractions) for instructional purposes. We propose enhancing Lesh's translational model with an immersive and interactive VR representational component (right).

The problem, however, of connecting the symbols to real-world situations remains; it is often difficult for students to integrate formal instruction with their informal knowledge. Mack [] suggests that comparison of fractions is 
sometimes difficult for students who regard fractions as discrete whole numbers rather than as proportions. For example, when comparing fractions such as $1 / 3$ and $1 / 4$, it is common for students to conclude that the fourth is larger than the third because four is a bigger number than three in the counting series. Students committing this type of error are probably applying knowledge of whole numbers to fractions. By relating the formal symbols to realistic situations and manipulative representations of fractional amounts, students may be less likely to consider the fourth as larger than the third. Similarly, Lesh and his colleagues, from their interviews with children, noted that children constructed what they refer to as informal strategies for ordering fractions []. These strategies reflect students' use of mental images of fractions to judge the fractions relative size and not taught procedures, such as least common denominators and cross-products.

Based on the above, we believe that a simulation-based environment, such as the kind provided by a VR environment, could provide an additional method of representation of such deep concepts that might aid in conceptual learning. This form of representation can combine the pictorial representation of fractions with a simulation of real-world situations and, in the case of interactive VR, the power of manipulative aids. Thus, we have enhanced Lesh's model with an immersive VR representational component (Figure 2) and have designed appropriate learning problems in an interactive virtual environment that involves tasks with fractions.

\subsection{The virtual environment: redesigning the layout of a playground}

We decided to incorporate learning problems based on fractions into an engaging VR application with a game-like scenario. Consequently, the idea of designing a playground emerged. We created both a Virtual Playground for a CAVE-like environment and a physical model using LEGO ${ }^{\mathrm{TM}}$ bricks. The tasks designed for the virtual playground application involve modifying the areas that the six main elements of the playground (swings, monkey bars, a slide, a roundabout, a crawl tunnel, and a sandpit) cover. Each element covers an area which is colour-coded and represented by blocks. The area representing each playground element is initially incorrect (either too big or 
too small) and must be redesigned, according to rules that require fractions calculations (Figure 3).
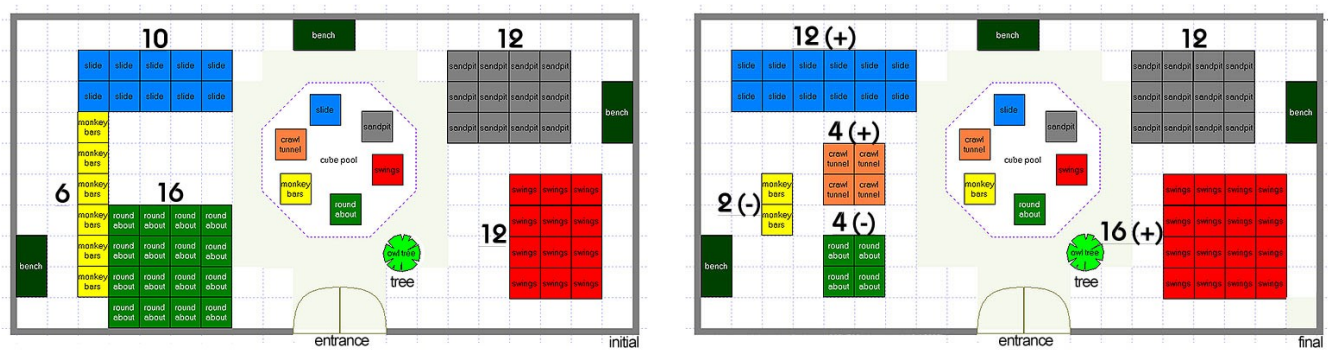

Figure 3. The footprint of the playground on the left image shows the initial layout of the playground; the footprint on the right illustrates one of the possible correct designs (green: roundabout, yellow: monkey bars, blue: slide, grey: sandpit, red: swings, orange: crawl tunnel).

The swings, for example, initially cover a $3 \times 4$ area, that is twelve blocks. The scenario requires that the area be increased by comparing two fractions (the fractions $1 / 3$ and $1 / 4$ ) and choosing the number that represents the larger amount. In this case, the fraction 1/3 which results in 4 blocks must be chosen and the 4 blocks must be added to the swings area, by picking blocks from the central pool and placing them on the 4 tiles that need to be covered.

\subsection{Scripting interactivity: system feedback mechanisms}

The system provides both direct and implicit visual and audio feedback to respond to the children's activity. The overall scenario and goal is presented to the participant by a virtual owl (Figure 4), while the rule for each area is provided by a coloured bird, which floats over that area and talks to the participant when clicked upon. The participant's interaction device (or "magic wand") includes a joystick for navigating the environment and three colourcoded buttons: the red button which allows the participant to switch between "block mode" (in which construction takes place) and "playground mode" (or review mode); the grey button which is used for picking and placing blocks and clicking on birds; and the blue button which is used to toggle between the default ground view and the top-down view of the playground.

When the participant constructs a correct area for an element (by either adding or removing blocks according to the rules), the 'red' button on the wand must be pressed in order to switch to "playground mode" and immediately see the playground element appear in place of the blocks. If the 
area is not formed correctly, then the playground element will not appear and the participant will be prompted to reconsider her actions. In addition to these methods of control over the environment, the system provides intrinsic feedback concerning placement of the blocks onto the playground tiles. For example, the system will not allow the participant to place a block next to the fence, near the benches, on the yellow-brick footpath or next to a block of a different colour. Visual and audio cues enhance these restrictions.
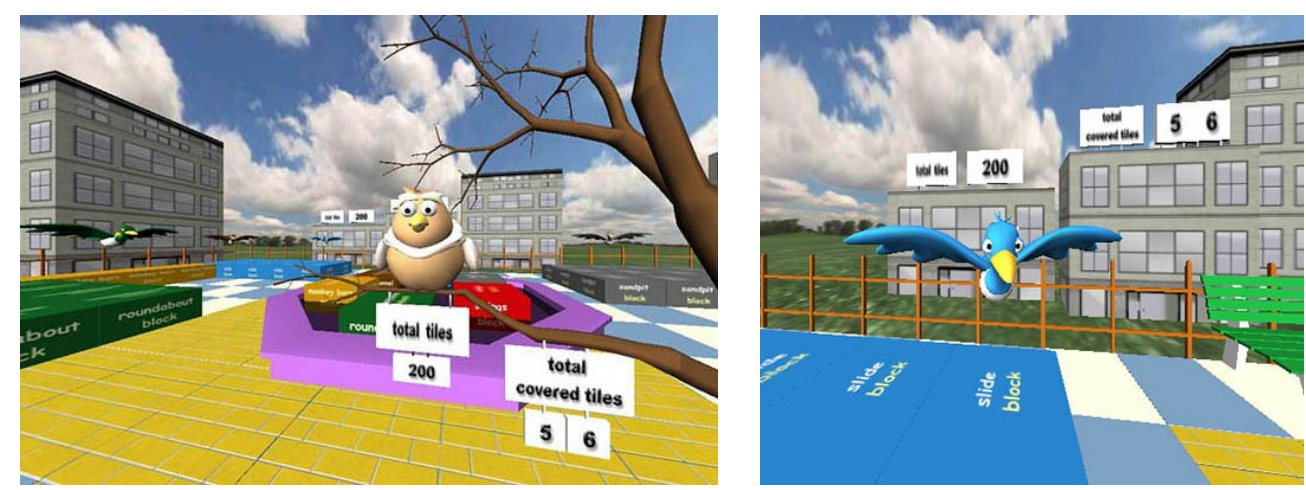

Figure 4. A view of the Virtual Playground, in which children re-design the layout of the playground based on rules provided by expressive virtual characters. The owl is the main character that greets each participant and provides the general rules before the participant starts the design game. Coloured birds speak out the rule for each area that needs to be changed.

It is important to note here that the Virtual Playground is not designed as an instructional environment following specific pedagogical models for teaching fractions, but as a tool for the evaluation of our research question concerning interactivity and learning. Hence, the characters (owl and birds) are neither avatars nor autonomous agents that respond intelligently to the participant's actions and questions. They are merely "rule providers", meaning that they simply state the rules of the tasks that must be performed (in place of a written instruction sheet, for example).

\section{Evaluation}

Empirical work was carried out with a total of 57 primary school students between the ages of 8 and 12, in different between-group experiments: an exploratory study, a pilot study, and a large-scale experiment. The exploratory study, as already described, aimed at defining the evaluation methodology and framework for analysis. The pilot study, which was carried out a few months prior to the main experiment, aimed at improving the usability of the 
$\mathrm{VE}$ and helped in organizing the overall process of the evaluation. The largescale experiment, which took place in 2005 , involved a total of fifty $(\mathrm{N}=50)$ children, 25 girls and 25 boys from different schools and socioeconomic backgrounds, who participated in one of three different conditions, two experimental VR conditions and a non-VR group.

\subsection{Experimental procedure}

Each study was conducted with one participant at a time lasting, on average, 90 minutes for each. The experimental methods included direct observation, interviews and pre- and post-test questionnaires, designed in collaboration with math teachers. Prior to the main activity, the participant was asked to fill out a questionnaire with math questions that are based on the fractions questions found in standardized tests (such as the Key Stage 2 SAT math test). A user profiling questionnaire was also given at this time. This included questions that attempted to draw a picture of the child's familiarity with computers, frequency of computer game play, and understanding of or prior experience with virtual reality.

\begin{tabular}{|l|l|l|l|c|c|}
\hline \multicolumn{1}{|c|}{ condition } & activity & interactivity & immersion & \multicolumn{2}{c|}{$\begin{array}{c}\text { participants } \\
\text { involved }\end{array}$} \\
\hline \multicolumn{2}{|l|}{} & \multicolumn{2}{|l|}{$\begin{array}{c}\text { female } \\
\text { male }\end{array}$} \\
\hline $\begin{array}{l}\text { C1: interactive } \\
\text { VR (IVR) }\end{array}$ & active & Yes & yes (VR cave) & 9 & 8 \\
\hline $\begin{array}{l}\text { C2: passive VR } \\
\text { (PVR) }\end{array}$ & passive & $\begin{array}{l}\text { no (watching a } \\
\text { robot interact) }\end{array}$ & yes (VR cave) & 5 & 9 \\
\hline $\begin{array}{l}\text { C3: non-VR } \\
\text { (LEGO) }\end{array}$ & active & No & no & 11 & 8 \\
\hline
\end{tabular}

Table 1. Condition attributes and numbers of participants involved.

After the questionnaire was completed, the child was assigned to one of three experimental conditions; either the non-VR condition or one of two VR conditions (Table 1). Each child participated in only one of the three conditions of the study (between-groups design). An even spread according to aptitude and gender was attempted; however the practical difficulties we encountered in recruiting the participants (one child at a time had to be 
brought to the virtual reality laboratory on a weekend) prevented us from achieving an equal number of boys and girls in each condition, although an equal number was achieved overall.
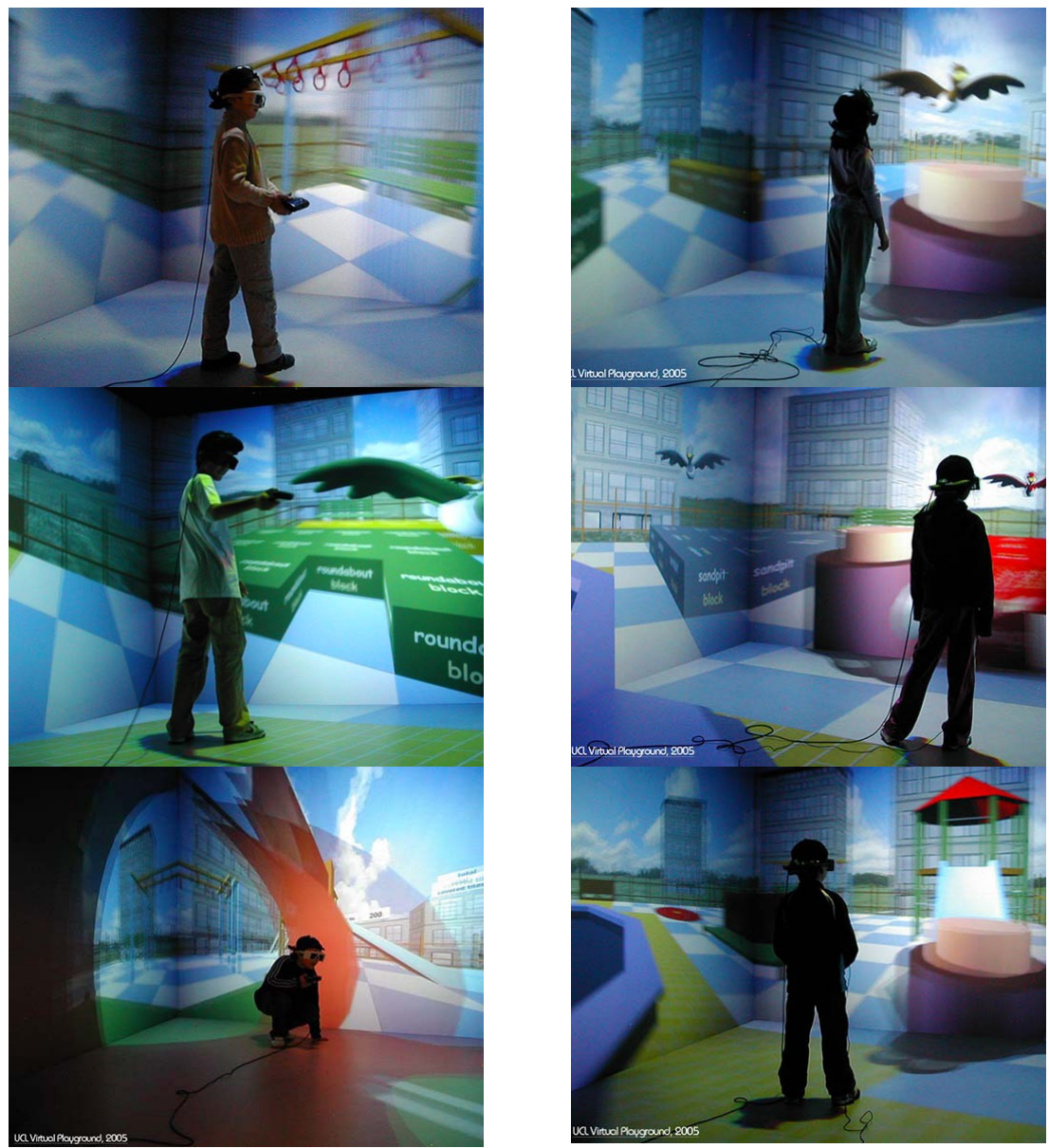

Figure 5. Images of children participating in the Virtual Playground studies, in the two experimental conditions: interactive VR condition (left column) and passive VR condition (right column).

The nature of the study was such that the student was free to act or interact for as long as she wished with the playground, be it the virtual or the non-virtual (LEGO) playground. A researcher who was at the same time the interviewer and the observer was constantly present, encouraging the participant to explain her/his actions while doing (by thinking aloud).

If assigned to the interactive VR experimental condition, the participant was immersed in a typical CAVE-like system ${ }^{2}$. The participant viewed the projected stereoscopic images by wearing a pair of active stereo glasses and could move around freely to interact with the environment by using a wireless wand which contains a joystick and buttons. The wand was used to navigate

\footnotetext{
${ }^{2}$ Consisting of four projection surfaces (three walls and the floor)
} 
around the virtual world, and to select and place virtual objects within that world, as described previously. A head tracker was specially adjusted on a cap that was worn by the participant, thus relaying the head position and orientation to the computer (Figure 5). Before starting, the task was explained to the participant who had a chance to practice navigating and moving objects around in the virtual space of a training environment.
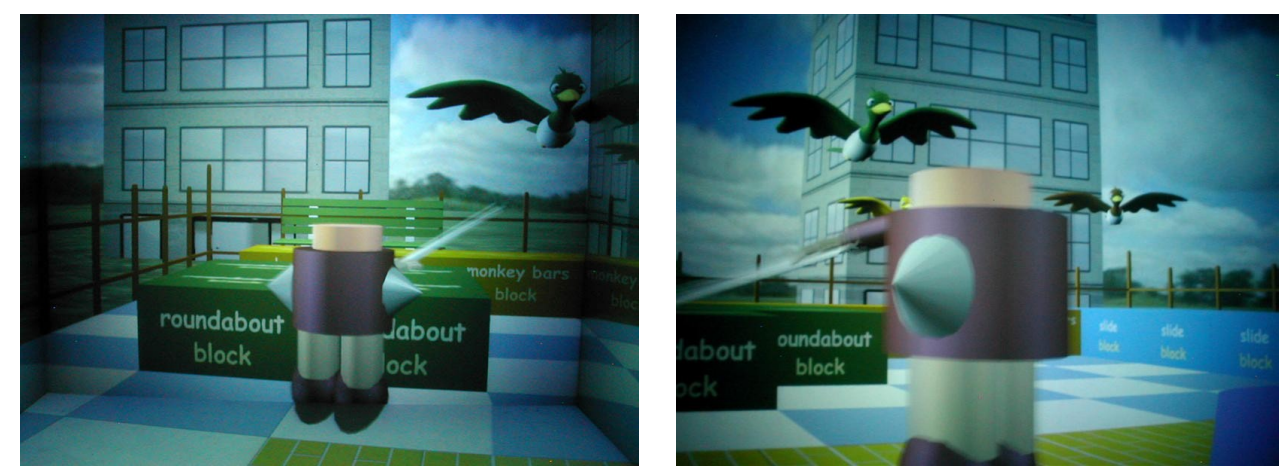

Figure 6. A robot character called "Spike" was used in the passive VR condition to play back a pre-recorded sequence of actions.

The second condition, the "passive VR" experience, took place in the same immersive environment; only, in this case, a pre-recorded sequence of actions involving the re-design of the playground was played out by a virtual character, a robot called "Spike" (Figure 6). The participant stood in the space wearing the stereoglasses and observed Spike as he went about listening to the rules and moving the blocks around as in a video sequence. The participant was encouraged to predict what Spike's actions would be ("what would you do if you were Spike?") and explain why Spike had done what he had done after each playground element was corrected.

Finally, if assigned to the non-VR condition, the participant took part in an activity using LEGO bricks (Figure 7). The activity involved the design of a playground on a grid-like floor plan, similar to seeing the playground from above in the virtual reality environment. As in the Virtual Playground, the differently coloured bricks represent the swings, slides, etc., which the participant must position according to the rules provided on cards. However, although each participant was actively involved in designing the playground, no response or feedback from the system existed. 

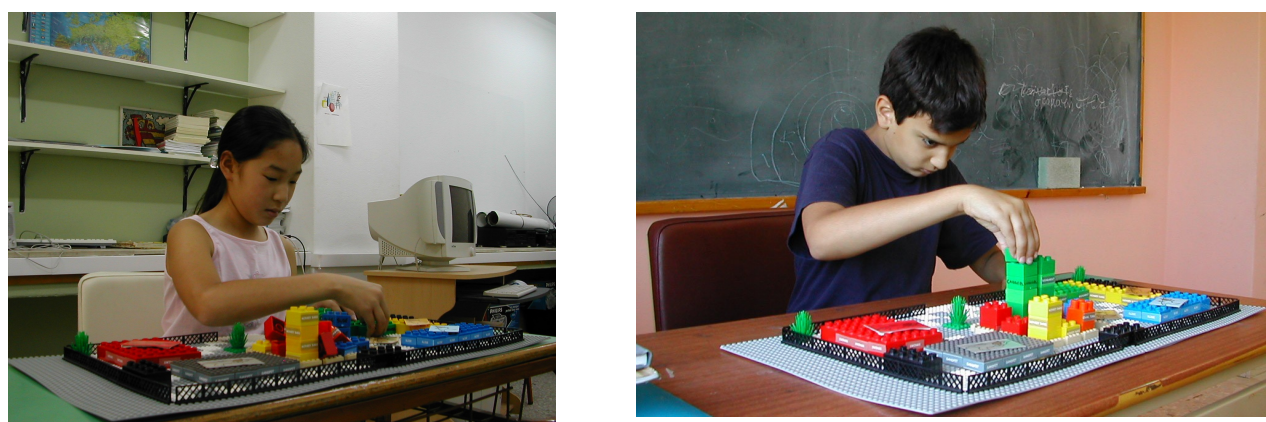

Figure 7. The non-VR condition involved redesigning the layout of the playground using LEGOTM $^{\text {TM }}$ bricks.

After the main experience was completed (activity in the interactive VR scenario, participation in the passive VR scenario, or activity with LEGO bricks), the participant was asked to complete a post-test with questions related to fractions, similar to the pre-test. Finally, every participant was interviewed about her experience by the observer, who noted the specific actions in which the participant had problems with, and directed the participant to reflect on these accordingly.

\section{Observations}

The studies have resulted in an enormous pool of data of multiple types, analyzed both quantitatively and qualitatively. The quantitative analysis showed no meaningful association between the different variables, such as gender, age, and condition, on student performance (measured through the pre- and post-tests). Therefore, for this paper we have chosen to present specific examples, extracted from the qualitative analysis, that provide us with interesting observations of student activity; instances of internal contradictions such as the ones that occurred during the analysis of the exploratory study involving column construction. The pool of data was reduced -selected and condensed into a manageable form- by means of an inductive analysis, which produced central themes and patterns that emerged during this analysis. The themes reported below have been chosen based on their being representative of typical experiences or learning problems. 


\subsection{The problem of comparing fractions}

A consistent finding in the study has been the confirmation of the difficulty that most children have when asked to compare fractions. Jack ${ }^{3}$, for example, is a 9 year-old boy who had scored very low on almost all of the questions in his pre-test. It was, thus, expected that he would have difficulty in the Virtual Playground with the swings task, which involved increasing the area of the swings (currently a $3 \times 4$ area of twelve blocks) by comparing two fractions (the fractions $1 / 3$ and $1 / 4$ ) and choosing the number that represents the larger amount. When the task was presented to him by the bird he immediately replied that he would increase the area by $1 / 3$. However, when asked by the observer how he came up with that result, in other words, how many blocks he believed that $1 / 3$ and how many $1 / 4$ represented, he replied that $1 / 4$ is four blocks and $1 / 3$ is five blocks. He then continued with his decision to add five blocks to the swings area. When he completed the placement of the blocks (inevitably creating a non-rectangular area), he clicked on the red button to switch to "playground mode" and see if his decision was correct. When he saw that it was not, he reflected on his construction and concluded that the area "did not have the right shape".

Lisa, a 10 year old girl who had been taught fractions in school and had average scores on her pre-test (Figure 8), made some decisions based on what "looked right". These decisions were evident in two cases, in which she made mistakes with her fractions. In the case of comparison between $1 / 3$ and $1 / 4$, she decided to increase the swings area by $1 / 4$. When asked why, she replied: "because I counted them and they are twelve, so divided by three they will not be enough... so... [I decided that it will be] four".

8. Observer: So you decided to increase by $1 / 4 \ldots$

9. Lisa: Yeah.

10. Observer: And how many blocks is that?

11. Lisa: Uhm... four.

Lisa made the common mistake (identified by []) of choosing $1 / 4$ as the fraction that results in the larger number. However, she correctly added four

${ }^{3}$ Pseudonyms have been given to all children that participated in the studies and that are mentioned here. 
blocks (the result of $1 / 3$, not $1 / 4$ ) to the swings area. This correct action seems, in part at least, to be attributed to her intuition (enhanced by the visual cues provided in the VE) rather than her calculations.
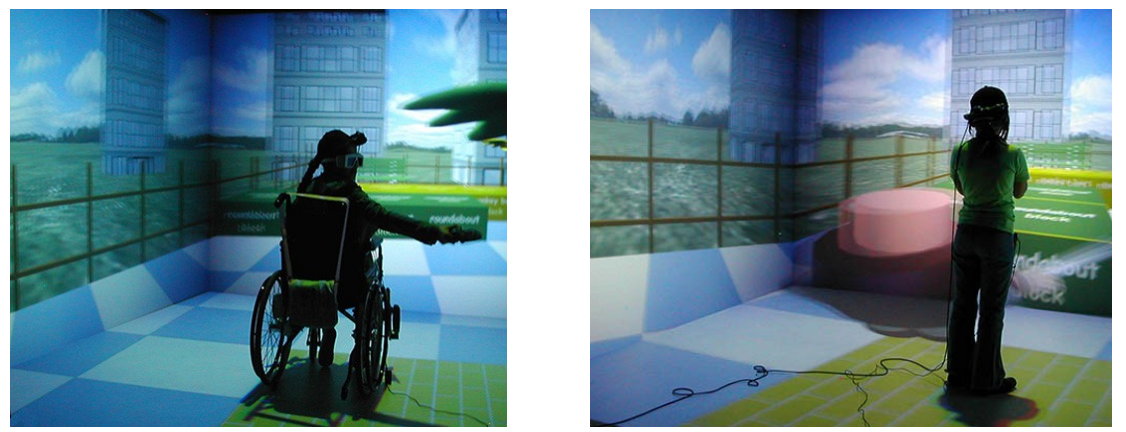

Figure 8. A 10 year-old girl (left) interacting with the Virtual Playground and a 9 year-old girl (right) observing the robot while he removes blocks from the playground.

\subsection{Response to system feedback}

Similarly to Lisa, Julie, a 9 year-old girl participating in the interactive VR condition, chose $1 / 4$ as the fraction that results in the larger number. However, unlike Lisa, Julie knew that $1 / 4$ of 12 results in 3 blocks and attempted to fit these three blocks in the correct place so as to complete the task. Julie tried out various solutions before realizing, through an approach of reflection that was guided by her recall of system feedback, that she should have chosen $1 / 3$ instead of $1 / 4$ :

12. Observer: Ok, so one third of twelve or one fourth of twelve is gonna give us more?

13. Julie: One fourth

14. Observer: How many blocks will one fourth give us?

15. Julie: So... [counts the blocks on the ground] ...there's twelve blocks... so, three.

16. Observer: So, where are you going to put these three blocks?

Julie clicks on her blue button to see the playground from above (Figure 9). In the top-down view she indicates where she plans to place the three blocks:

17. Julie: Two, either on this side... or no, I mean three blocks on around... this bit. 
18. Observer: Towards the fence or towards the sandpit?

19. Julie: Towards the fence.

She returns to ground view and attempts to place a block on the side of the swing area that is near the fence. This triggers the system feedback message: "This is too close to the fence".

20. Julie: Ok, so, on that side... but we can't do it on that side... I think I have to use the whole shape cause that's too close to the sandpit.

21. Julie: Ok, I know what I wanna do. I think. I'm going to bring... [thinking] ok, no, it's going to say "too close to the path..." cause if I put these three I think still it's gonna be too close... cause there's four here [meaning four free tiles] that might say the shape's not right...

22. Observer: You mean that it's not going to be a whole shape...

23. Julie: Wait, how much do I have to put, three or four? four! oh! we should do one third, cause one third of twelve gives us four and it'll complete a proper shape.

24. Observer: How come you didn't think about this from the beginning?

25. Julie: Cause the number four is bigger than three so it just came to my mind straight away.

26. Observer: You mean one fourth is...?

27. Julie: Just cause the number's bigger it just came to my head straight away.

Meanwhile, Julie has picked four blocks and has placed them one by one in the correct area.

28. Julie: Ok, red button!

Upon clicking on the red button, the model for the swings replaces the blocks that comprise that area, and Julie completes her task in the playground.
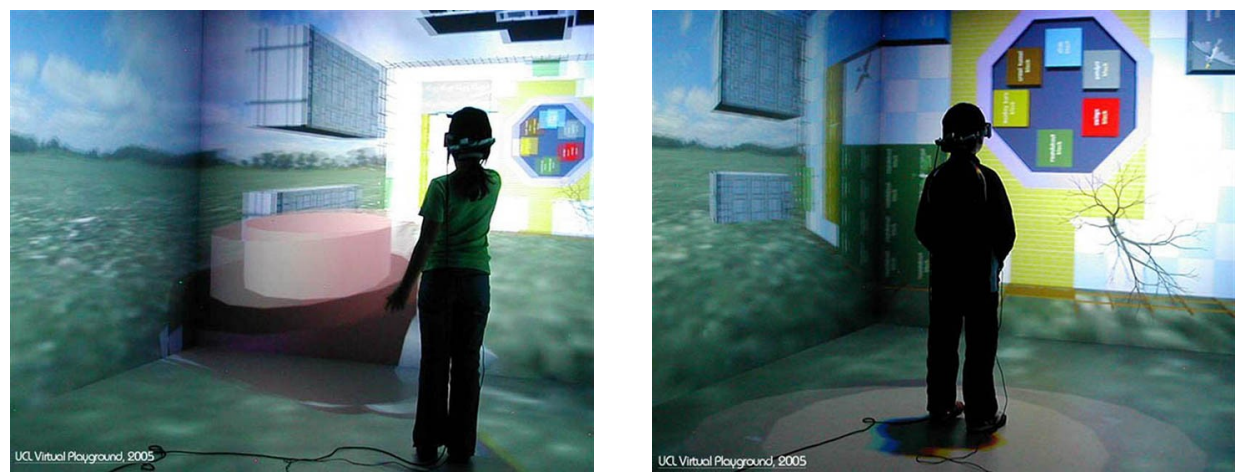

Figure 9. Children using the top-down view to plan the layout of the playground. 


\subsection{Substituting the denominator}

Another common mistake, made by more than half of the participants in the study, was the use of the denominator of a fraction as the resulting number required by the task. This problem was faced with two of the playground elements, the slide and the monkey bars, which involved tasks that required finding $1 / 5$ of 10 and $1 / 6$ of 12 respectively. For example, initially the monkey bars occupy an area of six blocks, placed in a long strip. The rule communicated to the participant states that the current area is too long and that it must be decreased by $1 / 6$ of the area of the sandpit (which occupies twelve blocks). David, an 11 year-old participant in the passive VR condition, immediately concluded that the correct answer is six.

29. Observer: What did the bird say?

30. David: That ...it's too long [the monkey bars] and that they have to be $1 / 6$ of the area of the sandpit...

31. Observer: How much is that?

32. David: Six.

He was certain that six was $1 / 6$ of twelve. However, the layout of the playground provoked an internal contradiction, since the monkey bars were already six blocks long, so if the robot took out six this would leave no blocks on the ground. When the robot removed four blocks leaving a total of two blocks on the ground and the blocks were correctly switched to monkey bars, David exclaimed that he had known all along that the correct answer was two but hadn't thought of it from the start. When asked later why he was confused even though he knew that $1 / 6$ of 12 is two, he responded that the correct result (two blocks) did not make sense to him, because "in real life the area for the monkey bars could not have been so short". However, after seeing Spike (the robot) performing the task, he was able to explain why the correct answer was two blocks.

Cherry, a confident and very talkative 9 year old girl who participated in the passive VR condition, had a similar response to the slide task (which involved increasing the existing area of 10 blocks by one fifth). As soon as the blue bird finished presenting the rule for the slide, Cherry began counting aloud in order to direct Spike on what to do: 
33. Cherry: One two three... one two three four five six seven eight... So it said that it's covered... one fifth... one fifth... is what it's supposed to be.

34. Observer: It has to be one fifth more of what it is now. So, how many blocks are there now?

35. Cherry: Ten.

36. Observer: Ok. So how many would you add if you were Spike?

37. Cherry: Five.

38. Observer: Spike has started already trying to add blocks. Where would you add those blocks?

39. Cherry: There [showing the row of five tiles near the crawl tunnel]. Mmm, no... there [pointing near the footpath]... mmm no... can you put them on the yellow road?

40. Observer: I don't know. Well look at Spike and tell me what you think he's doing.

41. Cherry: Oh, he's putting it over there [pointing at the other side of where she was thinking the blocks should go].

42. Observer: Ok, so how many blocks does he need to put?

43. Cherry: Two.

44. Observer: You said five before.

45. Cherry: No I mean there [showing the two tiles where the robot was already putting the first block].

46. Observer: So finally how many blocks does he have to put to make this one fifth bigger of what it is?

47. Cherry: Five.

48. Observer: So, he put one already. Where is he putting the second one?

49. Cherry: Next to the first one.

50. Observer: How about the others?

51. Cherry: Uhm, down there. Down on the left side [showing the row of five tiles she had shown originally, next to the crawl tunnel]... No, uhm, on that bit [showing the two tiles on the footpath]

52. Observer: How many more does he need to put? 
53. Cherry: Three.

54. Observer: What if he clicks on his red button now to see what happens? [The robot "clicks" on the red button and the blue blocks turn into a slide].

55. Cherry: Oh! So it only needed two.

56. Observer: Do you know why?

Cherry shakes her head in a 'no' motion.

57. Observer: So you don't understand why, do you remember (what had to be done)?

58. Cherry: So there's two... [thinking for a few seconds]. Ten, so... oh yeah, you have to times it by two to get twenty.

59. Observer: Twenty?

60. Cherry: No I mean divide. Divide ten by two and you get... uhm... five... yeah what he did. No, no divide ten by five and you get... ten by five... two. Yeah, that's what he did.

Cherry finally is able to explain how the number two was derived as the correct answer. As Kuuti [] notes, initially each operation is a conscious action, consisting of orientation, i.e. planning in the consciousness by using a model, and execution phases. When, however, the corresponding model is good enough or the action has been practiced long enough, the orientation phase will fade and the action will be collapsed into an operation. Indeed, in Cherry's case, a phase of conscious planning took place when she was originally asked to identify how many blocks she would add to the slide area if she were Spike. An execution phase followed where she showed where she would place the five blocks she had identified as being the correct answer for fixing the slide area. However, when Spike completed the slide area correctly by placing only two blocks, a contradiction occurred between Spike's action and Cherry's model. Cherry had to question her model and drastically change it as it proved to be incorrect. Using a kind of "backward thinking" process to explain why the correct answer was such and resolve the contradiction, she came up with a new model (in which the original number of blocks is divided by the denominator) that could later be generalized. In fact, in the next task, which was to compare the two fractions for increasing the area of the swings, she used her newly constructed model to come up with a correct response 
immediately. The form of her explanation of how the correct answer was derived indicates that the previous action of correcting the slide has become fluent, turning into an operation. So, as the red bird finished telling the rule (which required increasing the swings area, now consisting of twelve blocks, by one third or one fourth, whichever gives more blocks), Cherry started counting:

61. Cherry: One, two, three, four... [counting the blocks of the swings] ...twelve. So did she [the red bird] say one fourth?

62. Observer: She said one third or one fourth, whichever gives you more blocks.

63. Cherry: One third [with certainty].

64. Observer: How many blocks does that give you?

65. Cherry: Four.

66. Observer: So how did you find that?

67. Cherry: Twelve divided by three.

68. Observer: And how much does one fourth give you?

69. Cherry: One fourth... three...

70. Observer: Ok, so between the two which gives you more blocks?

71. Cherry: One third... yeah.

As the orientation phase is clear right away, the observer continues by asking about the execution phase. When the robot has finally placed all four blocks and is ready to click on the red button, Cherry is asked if she thinks what the robot had done was right. She responded yes with certainty and her response was confirmed by the appearance of the swings. According to Kuuti, this kind of action-operation dynamic is a fundamentally typical feature of human development. For an individual to become more skilled in something, operations must be developed so that someone's scope of actions can become broader as the execution itself becomes more fluent []. The question posed by this research is whether the interactive properties of a VE, e.g. cues and system feedback, can enable this transformation from conscious actions into operations, where planning and problem solving will have faded from the consciousness. 
In summary, the examples presented above reinforce our view that some decisions were made intuitively, supported by the visual cues provided by the environment (the shape of each area and the surrounding space), and the feedback mechanisms programmed into the system. These cues and feedback aided some children at solving the learning problems, suggesting that their intuitive action may be closely linked to the form of the representation of the problems and, consequently, the value of VR over formal, abstract instruction as a way of supporting learning.

\section{Discussion}

During the first sets of studies (the exploratory studies concerning column construction and the pilot studies with the Virtual Playground), a number of methodological and practical issues emerged related to the challenges of designing and evaluating technology for and with children. For the main studies, the focus was to capture behavioral and conceptual change, which can lead to indications of learning triggered by interactive activity in the virtual environment. To identify this change a number of measures were taken. Different conditions resulted in a between-groups design, attempting to cover the different combinations of activity, interactivity and immersion. Then, multiple different methods of testing were designed, ranging from the quantifiable pre- and post- questionnaires to the more qualitative observations and interviews. This was to ensure that the data collected would result in a wealth of information, which we could meaningfully combine and analyze.

The quantitative analysis did not provide evidence that interaction has any effect on children's ability to learn fractions. On the other hand, the qualitative analysis seemed to be more appropriate at describing the richness of interaction between the multiple factors that came into play in this study. The use of an analytical framework such as Activity Theory provided the lens through which we were able to identify the critical incidents and internal contradictions - conflicts that required further attention as possible indications of conceptual change. Hence, some generalizations emerged from the analysis of the different cases, especially when examining each child's activity and reaction to individual problems. Within each case, we identified the 
individual sections, or instances, where interesting contradictions occurred, and related these to the other measures (scores on the tests and especially recall of activity during interview discussions). The examples that were presented here seem to suggest that the actions based on the implicit cues (getting the shape of the area right, for instance) or on the feedback provided by the virtual environment (taking into account the restrictions in placing the blocks on certain tiles) helped most students complete the tasks successfully. However, there was no evidence that successful problem solving in the interactive VR condition resulted in their understanding of the underlying concept, nor did it demonstrate proof of conceptual change on a deep level. If anything, it was the passive VR condition that proved to be surprisingly interesting in that it fostered a certain kind of reflective process on the part of the student (e.g., as shown from Cherry's interaction). All of the children who participated in the passive VR condition enjoyed watching and verbally directing the robot in performing the tasks. After completion of each task, the student was prompted by the observer to explain what the robot had done and why. For the children that had difficulties with the tasks, the robot seemed to take on the role of a more able peer, essentially demonstrating the correct answer. In this sense, the passive VR condition provided, implicitly, a guided form of experience, where the learner embarked in a process of reflective observation (watching others or developing observation about own experience []). The robot acted as an additional level of mediation which seemed to support the children's reflective thought, the ability to step back and consider a situation critically and analytically, with growing awareness of their own learning process. This finding agrees with the Vygotskian view that learning environments should involve guided interaction, permitting children to reflect on inconsistency and to change their conceptions []. It also suggests that perhaps a learning environment that combines guided activity with an enhanced prompting mechanism on behalf of the system may be more effective in fostering a reflective process that can lead to conceptual change. On the other hand, a fully interactive environment such as the one provided for the IVR condition in this study, although beneficial in problem solving, may be lacking the necessary support to scaffold conceptual learning. 
The examples presented here represent a very small view of the data that has been collected and the subsequent issues that emerge. A host of factors can influence learning, especially in sensory rich environments such as immersive virtual environments. Thus, further work is required before we can explain the elements that comprise the complex relationship between the learner, the tool (VE) and the learning objective, and derive more precise evidence of cognitive outcomes. Nevertheless, as the potential of immersive virtual reality for conceptual learning remains high and its deployment in public spaces continues to increase, study must continue if we are to acquire a deeper understanding of what constitutes learning within virtual environments.

\section{Acknowledgements}

The authors wish to thank all the children that participated in the studies and their parents, as well as the educators and researchers that volunteered to help with the design of the learning content, the long recruitment process, and the validation of observations and interpretations. Special thanks are due to Maria Mplouna, mathematics teacher, for the time, enthusiasm, and expertise that she has offered to this project and to Alexandre Mangon-Olivier, Dimitris Christopoulos, and George Drettakis for modeling, animation and technical guidance concerning the implementation of the virtual environment. The studies for this research have been approved by the UCL Committee on the Ethics of Non-NHS Human Research, Study No. $0171 / 001$.

\section{References}

1. Winn, W., Windschitl, M., Fruland, R., \& Lee, Y. (2002). When does immersion in a virtual environment help students construct understanding? In Proceedings of International Conference of the Learning Sciences, Seattle, Washington, USA, pp. 497503.

2. Waterworth, E. L., \& Waterworth, J. A. (2000). Presence and Absence in Educational VR: The Role of Perceptual Seduction in Conceptual Learning. Themes in Education, $1(1), 7-38$.

3. Jelfs, A., \& Whitelock, D. (2000). The notion of presence in virtual learning environments: what makes the environment "real". British Journal of Educational Technology, 31(2), 145-152.

4. Arthur, E., Hancock, P. A., \& Telke, S. (1996). Navigation in virtual environments. In Proceedings of SPIE - The International Society for Optical Engineering, Orlando, FL, USA, pp. 77-85. 
5. Slater, M., Steed, A., McCarthy, J., \& Maringelli, F. (1998). The Influence of Body Movement on Subjective Presence in Virtual Environment. Human Factors, 40(3), 469477.

6. Winn, W. (2005). What We Have Learned About VR and Learning and What We Still Need to Study. In Proceedings of Virtual Reality International Conference Laval Virtual, Laval, France, pp. 8-17.

7. Byrne, C. M. (1996). Water on Tap: The Use of Virtual Reality as an Educational Tool. PhD Dissertation, College of Engineering, University of Washington, Seattle.

8. Wiig, E. H., \& Wiig, K. M. (1999). On Conceptual Learning (Working Paper 1999-1 No. KRI WP 1999-1): Knowledge Research Institute, Inc.

9. Pares, N., \& Pares, R. (2001). Interaction-Driven Virtual Reality Application Design. A Particular Case: El Ball del Fanalet or Lightpools. PRESENCE: Teleoperators and Virtual Environments, 10(2), 236-245.

10. Johnson, A. E., Moher, T., Ohlsson, S., \& Gillingham, M. (1999). The Round Earth Project: Collaborative VR for Conceptual Learning. IEEE Computer Graphics \& Applications, 19(6), 60-69.

11. Allison, D., Wills, B., Bowman, D. A., Wineman, J., \& Hodges, L. F. (1997). The Virtual Reality Gorilla Exhibit. IEEE Computer Graphics and Applications, 30-38.

12. Roussou, M. (2000). Immersive Interactive Virtual Reality and Informal Education. In Proceedings of $i 3$ spring days, workshop on User Interfaces for All: Interactive Learning Environments for Children, Athens, Greece.

13. Kaufmann, H., \& Schmalstieg, D. (2003). Mathematics and Geometry Education with Collaborative Augmented Reality. Computers \& Graphics, 27(3), 339-345.

14. Dede, C. J., Salzman, M. C., \& Loftin, B. R. (1996). MaxwellWorld: Learning Complex Scientific Concepts Via Immersion in Virtual Reality. In Proceedings of Second International Conference of the Learning Sciences, pp. 22-29.

15. Dede, C. J., Salzman, M. C., \& Loftin, B. R. (1996). ScienceSpace: virtual realities for learning complex and abstract scientific concepts. In Proceedings of Virtual Reality Annual International Symposium (VRAIS 96), pp. 246-252.

16. Rose, H. (1995). Assessing Learning in VR: Towards Developing a Paradigm Virtual Reality Roving Vehicles (VRRV) Project. (Technical Report TR-95-1): Human Interface Technology Laboratory, University of Washington.

17. Bricken, M., \& Byrne, C. M. (1993). Summer students in VR: a pilot study. In Virtual reality: applications and explorations (pp. 178-184): Academic Publishers Professional.

18. Barab, S. A., Hay, K. E., \& Barnett, M. G. (1999, April). Virtual Solar System project: Building Understanding through model building. In Proceedings of Annual Meeting of the American Educational Research Association, Montreal, Canada.

19. Roussou, M., Johnson, A. E., Moher, T. G., Leigh, J., Vasilakis, C., \& Barnes, C. (1999). Learning and Building Together in an Immersive Virtual World. PRESENCE: Teleoperators and Virtual Environments, 8(3), 247-263. 
20. Moher, T., Johnson, A. E., Ohlsson, S., \& Gillingham, M. (1999). Bridging Strategies for VR-Based Learning. In Proceedings of ACM SIGCHI 1999 (CHI '99: Conference on Human Factors in Computing Systems), Pittsburgh, PA, pp. 536-543.

21. Otero, N., Rogers, Y., \& du Boulay, B. (2001). Is Interactivity a Good Thing? Assessing its benefits for learning. In Proceedings of 9th International Conference on HCI, New Orleans, pp. 790-794.

22. Steuer, J. (1992). Defining Virtual Reality: Dimensions Determining Telepresence. Journal of Communication, 42(4), 73-93.

23. Nardi, B. A. (Ed.). (1996). Context and Consciousness: Activity Theory and HumanComputer Interaction. Cambridge, Massachusetts: MIT Press.

24. Roussou, M. (2003). Examining Young Learners' Activity within Interactive Virtual Environments: Exploratory Studies (Technical Report No. RN/04/08). London, UK: University College London, Department of Computer Science.

25. Mack, N. K. (1990). Learning fractions with understanding: Building on informal knowledge. Journal for Research in Mathematics Education, 21, 16-32.

26. Cramer, K., Behr, M. J., Post, T. R., \& Lesh, R. (1997). Rational Number Project: Fraction Lessons for the Middle Grades. Dubuque, Iowa: Kendall/Hunt Publishing Co.

27. Harel, I. (1991). Children Designers. Interdisciplinary Constructions for Learning and Knowing Mathematics in a Computer-Rich School. Norwood, New Jersey: Ablex Publishing Corporation.

28. Kafai, Y. B. (1995). Minds in play: Computer game design as a context for children's learning. Hillsdale, NJ: Lawrence Erlbaum Associates.

29. Lesh, R., Landau, M., \& Hamilton, E. (1983). Conceptual models in applied mathematical problem solving research. In R. Lesh \& M. Landau (Eds.), Acquisition of Mathematics Concepts \& Processes (pp. 263-343). NY: Academic Press.

30. Kuuti, K. (1996). Activity Theory as a potential framework for human-computer interaction research. In B. A. Nardi (Ed.), Context and Consciousness: Activity Theory and Human-Computer Interaction (pp. 17-44). Cambridge, Massachusetts: MIT Press.

31. Kolb, D. A. (1984). Experiential Learning: Experience as the Source of Learning and Development. Englewood Cliffs, N.J.: Prentice-Hall, Inc.

32. Vygotsky, L. S. (1978). Mind in Society: The Development of Higher Psychological Processes. Cambridge, MA: Harvard University Press. 